\title{
Understanding of the Coherent Demodulation with Phase-Locked Loop
}

\author{
Bingcong Zhai ${ }^{1}$ \\ ${ }^{1}$ School of Information Engineering Beijing, Institute of Petrochemical Technology, North Qingyuan Road, Daxing District, \\ Beijing, China
}

\begin{abstract}
The phase-locked loop (PLL) technology is a very important technology in the communication field. With the development of electronic technology toward digitalization, the phase-locked processing of signal needs to be realized in digital way. Therefore, more and more attentions have been paid to the research and application of all digital phase-locked loops. This paper serves as an introduction about the basic background of PLL, the basic characteristics and structure of PLL, and the basic principles of modulation and demodulation. It provides a concise application about the basic principle and main design process of modulation and demodulation of FSK signal, which are realized by using phase-locked loop chip NE564.
\end{abstract}

\section{INTRODUCTION}

In 1932, De Bellescize invented the first phase-locked loop and presented the theory of synchronous detection. People were constantly exploring the application of PLL technology. Until 1970s, because of the rapid development of integrated circuit technology, the integrated phase-locked loop technology gradually rose. The cost of phase-locked loop gradually was reduced and the function was gradually improved, which provided widely space for PLL technology application development. Up to now, PLL has been widely applied in communication, radar, instrumentation, spaceflight, navigation, computer, industry, geology and other technology fields.

With the development of digital electronic technology, the digital phase-locked loop (PLL) is also rising. A large number of digital phase locked loops are widely used in the fields of digital carrier signal transmission synchronization, bit synchronization, coherent demodulation and so on. But the start appearance of the digital phase locked loop is a half analog circuit which still has some analog devices in the circuit. The analog devices will be influenced by temperature and voltage. In order to solve these problems, an all-digital phase-locked loop technology has appeared. All-digital phase-locked loop has high accuracy and is not affected by voltage and temperature. It can preset the central frequency and loop bandwidth of phase-locked loop and is easy to construct high-order phase-locked loop. With the development of integrated circuit technology, the frequency of PLL is increasing and the whole system can be made into system on chip. The phase-locked loop circuit is made into a function IP core, which is embedded in SOC to form in chip phase locked loop. At the same time, with the rapid development of Field - Programmable Gate Array and Complex Programmable Logic Device technology, the performance index of an all-digital phase-locked loop have also been rapidly developed and greatly improved.

Although the development of PLL technology has more than 80 years, the application of phase-locked loop technology is constantly expanding. Because of the development of the high-tech product, People need to improve the index of PLL. So many domestic and foreign engineers in the circuit design of the phaselocked loop technology continued their research to get the new PLL structure which can be satisfied in different occasions. This paper is going to talk about the coherent demodulation with phase-locked loop. The first section is the structure of PLL. The second section is the introduction of modulation and demodulation. The third section is the application about the FSK signal using PLL chip.

\section{BACKGROUND}

PLL is the fusion of automatic frequency control and automatic phase control technology. In 1930s, scientists built synchronous control theory and the French engineer Bellescize published a mathematical description of PLL and Synchronous detection theory in 1932. But the circuits were complex and the cost was high. Thus, the PLL was not widely available. In 1943, the phase-locked loop was firstly used in black and white TV receiver. It can suppress the external noise which could disturb the synchronous signal. Then, the phase-locked loop circuit was used to synchronize the color pulse string in the color TV receiver. Since then, the phase-locked loop has

*Corresponding author: 18601167058@163.com 
been applied and developed rapidly.

In 1950s, with the development of space technology, Jaffe and Rechtin successfully used phase-locked loop filter as the tracking missile beacon. It was the first time that they published the article about the analysis of the linear theory of phase-locked loop on noise effect and solved the problem about optimal design of phase-lock loop. The development of space technology promoted the further discussion of the phase-locked loop and its theory and greatly promoted the development of phase locked technology.

In the early $60 \mathrm{~s}$, Viterbi studied the nonlinear theory of the noiseless phase-locked loop and published a paper about the principle of coherent communication. With the development of semiconductor technology, the phaselocked loop chip has been widely applied in various fields as a low-cost multi-functional component.

In 1970s, Undsy and Chanes researched the nonlinear analysis theory of noise of one order, two order and high order PLL. With the extensive research on the theory and application of phase-locked technology and the development of digital circuit, the phase detector was partly replaced by digital circuit and the rest part was still analog circuit, which was the first digital phaselocked loop. The exact name of it was the Mixed-single PLL. With the continuous development and perfection of the digital mode, Hybrid PLL technology and theory became the mainstream of the phase-locked loop.

Now, with the demand of low cost, low power consumption and high data transmission rate, the integrated circuit is developing towards the direction of high integration and low power consumption. Phaselocked loop technology has become a systematic theoretical science. It has been widely applied in communication, radar, aerospace, precision measurement, computer, infrared, laser, atomic energy, stereo, motor control and image technology departments.

America nation Semi-Conductor announced launching the LMx243x series phase-locked loop chip in June 2003. Its operation frequency was higher than $3 \mathrm{GHz}$. It was suitable for WLAN, $5.8 \mathrm{GHz}$ indoor cordless phone, mobile phone and base station. Low power and ultra-low phase noise (the normalization phase noise can reach $-219 \mathrm{dBcmz}$ ) makes it prominent.

In China, Haokai Microelectronics (Shanghai) Co. Ltd research and development high performance clock phase-locked loop which own completely independent intellectual property rights. Now the series of products have been pass the Multi Project Wafer silicon verification. The PLL with a new structure, unique and differential charge pump Voltage Controlled Oscillator design, can make ensure that PLL has very low noise. The unique design of the differential VCO can maintain the $50 \%$ duty cycle of the output clock. Power consumption can be reduced to $1 / 4$ of conventional design.

\section{STRUCTURE OF PLL}

In this section, we will talk about the structure of PLL.PLL is a feedback circuit, which can make one signal to track another one. Since PLL has a tricking function, it is usually used in closed loop tracking circuit. When the frequency of output signal is equal to the frequency of input signal, the output voltage and input voltage maintain a fixed phase difference. It means that the phase of output voltage and input voltage is locked, which is the origin of phase-locked loop name. PLL keeps an output signal synchronizing with a reference input signal in frequency as well as in phase. It is widely applied in the fields of communication, radar, measurement and automatic control.

The PLL in Fig 1 consists of a phase detector (PD), a loop filter (LF), and a voltage-controlled oscillator (VCO). This structure is just like driving cars. When we drive on the road, our eyes like a discriminator, which has the same function as phase detector. Our eyes responsible for distinguishing the direction of the car (feedback) and the road ahead (input) whether there is a difference. And then our brain judges the difference and command the hands rotate the steering wheel to make sure our car going straight. In this construction direction, the car is like the feedback and the loop filter and the voltage-controlled oscillator are like our brain to filter some useless signal and deal with some useful signal.

Firstly, we discuss about the Phase detector. The function of Phase detector is like an institution of phase monitor, which can find the phase difference between the input signal and the feedback signal and then expressing the difference in a reasonable way. This difference can be expressed by voltage form. The function of the relationship between the phase difference and the output voltage is called Phase discrimination characteristics. Phase detector is one of the basic components of phase-locked loop.

The characteristics of the Phase detector are expressed in $U_{d(t)}=K_{d} f\left[\theta_{e(t)}\right] . K_{d}$ is the gain coefficient of the phase detector and $\theta_{e(t)}=\theta_{1(t)}-\theta_{2(t)}$. This function indicates the phase difference between the two input signals. The function $f(x)$ represents the Phase discrimination characteristics, which reflects the relationship between the output voltage of the phase detector and the phase difference. The common characteristics of phase discrimination are cosine, sawtooth and triangle and so on. If the input is $X_{i}$ and the VCO signal is $X_{0}$, the expressions will be as $X_{i(t)}=$ $A \cos \left(w_{i t}+\theta_{i}\right)$ and $X_{0(t)}=B \cos \left(w_{0 t}+\varphi_{0}\right)$ where and are angular frequencies of the input signal and the VCO; and are their phase constants. If the loop is initially unlocked and the phase detector has a sinusoidal characteristic, the significant output signal at the PD is given by $V_{e(t)}=K_{d}\left\{\cos \left[\left(w_{i}-w_{0}\right) t+\theta_{i}-\varphi_{0}\right]+\right.$ $\left.\cos \left[\left(w_{i}+w_{0}\right) t+\theta_{i}+\varphi_{0}\right]\right\}$, where $K_{d}$ is the gain of the PD.

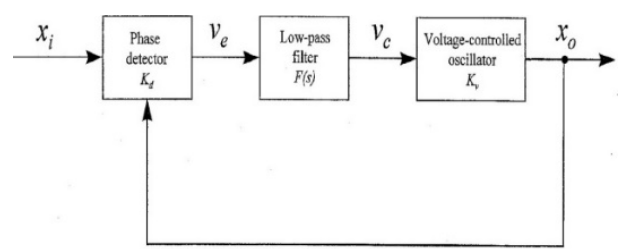

Fig 1 Basic topology of the PLL 
Secondly, we talk about the loop filter. In fact, loop filter is one type of filters, which is used in the loop circuit. Therefore, people name it loop filter. It is an important part of the PLL circuit. In order to understand it, you must firstly understand the principle of PLL. The low-pass filter has two functions: one is that the loop circuit can suppress high frequency error components at the output of the Phase detector, which improves the anti-jamming performance. The other is that when the loop filter is out of lock state, the loop filter can be stored in a short time and recover the signal quickly.

A loop filter is usually a linear circuit that consist of a linear element resistance, a capacitor and an operational amplifier. The loop filter is used for attenuation phase error caused by the input signal noise and high frequency components leaked by a smooth phase detector. The order of the loop filter and the noise bandwidth determine the dynamic response of the loop filter.

Last but not least, we talk about voltage-controlled oscillator. The definition of voltage-controlled oscillator (VCO) is the unit controlling the corresponding relationship between the output frequency of the oscillation circuit and the input control voltage. Its frequency equals to the function of input voltage. The work condition of $\mathrm{VCO}$ or Component parameters of oscillation circuit is influenced by the input control voltage. The characteristics of VCO is represented by the relation curve between the output angle frequency and the input control voltage $U_{c}$ (Figure 1). In the diagram, the angular frequency of $U_{c}$ at zero is called the free oscillation angle frequency and the slope of the curve of is called the control sensitivity. In a communication or measuring instrument, the input control voltage is a signal intending to be transmitted or desired. The voltage-controlled oscillator is usually called a fequency modulation by the most of people, which is used to produce FM signals. In the automatic frequency control loop and the phase-locked loop, the input control voltage is the error signal voltage and the voltage-controlled oscillator is controlled parts in the loop.

The types of VCO are LC voltage-controlled oscillator, RC voltage-controlled oscillator and crystal voltage-controlled oscillator. The VCO requires good frequency stability, high control sensitivity, wide FM range, linear relationship between frequency offset and control voltage, suitable for integration and so on. The crystal voltage-controlled oscillator has high frequency stability while the FM range is narrow. The frequency stability of RC VCO is lower and the FM range is wide. The characteristic of LC voltage-controlled oscillator between the crystal voltage-controlled oscillator and the RC VCO.

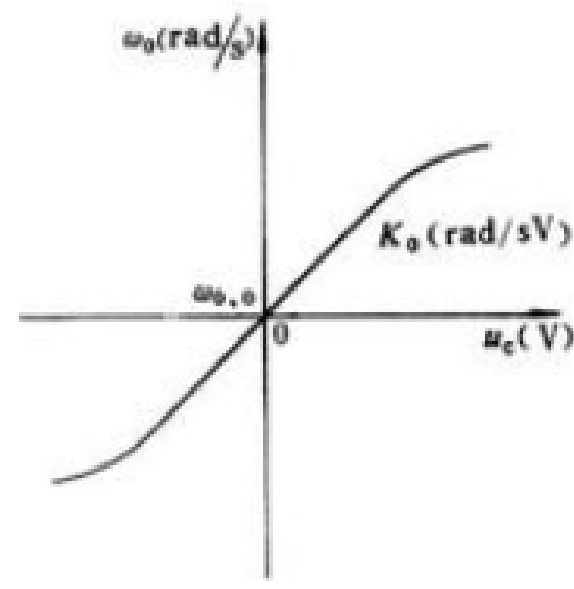

Fig 2 control characteristics of a voltage-controlled oscillator

\section{MODULATION DEMODULATION}

AND

In order to realize long-distance information transmission, people usually used to modulate the signal at the transmitter. After receiving the signal, the receiver will demodulate to restore the original signal. In fact, the modulation is a parameter which can carry the information of input signal to control the carrier signal. The parameters of the carrier signal have amplitude, frequency and phase, so there are three kinds of ways to modulate the signal: amplitude modulation (AM), frequency modulation (FM) and phase modulation (PM).

The characteristics of amplitude modulation wave is that the frequency- is equal to the frequency of the carrier signal and the amplitude varies with the change of the amplitude of the input signal. The characteristics of FM wave is that the amplitude is equal to the amplitude of the carrier signal and the frequency varies with the change of the amplitude of the input signal. The characteristic of phase modulation wave is that the amplitude is equal to the amplitude of the carrier signal and the phase varies with the change of the amplitude of the input signal.

Demodulation is the inverse of modulation, which can restore the modulation wave into the original signal.

There are many types of modulation and the classification methods that are not consistent. In the form of modulated signal, it can be divided into analog modulation and digital modulation. The process using analog to modulation is called analog modulation. And the process using data or digital modulate signal is called digital modulation. Corresponding to the classification of modulation, the demodulation can be divided into sine wave demodulation (sometimes also called continuous wave demodulation) and pulse wave demodulation. Sine wave demodulation can also be divided into amplitude demodulation, frequency demodulation and phase demodulation.

According to the modulation method, there are two types: linear modulation and nonlinear modulation. Linear modulation includes amplitude modulation (AM), suppression of carrier amplitude modulation (DSB-SC), 
band amplitude modulation (SSB), residual band amplitude modulation (VSB) and so on. The nonlinear modulation of anti-interference performance is stronger than linear modulation and the nonlinear modulation includes frequency modulation (FM), frequency shift keying (FSK), phase shift keying (PSK), differential phase shift keying (DPSK) etc. The linear modulation will not change the original signal spectrum structure, while the nonlinear modulation.

Coherent demodulation is also called synchronous demodulation and it is suitable for all the demodulation of linear modulation signals. The key to realize the coherent demodulation is to restore a coherent carrier that is strictly synchronized with the modulation carrier. Coherent demodulation usually uses a multiplier to multiply the input a reference signal that is coherent with the carrier frequency and the carrier frequency.

\section{APPLICATIONS}

In this part, we discuss the design of modulation and demodulation circuit for FSK signal using PLL chip.

Firstly, we talk about the Phase-locked loop chip NE564 and CD4046. High frequency analog phaselocked loop NE564 is produced by Philips Semiconductor (Holland Philip Company). The highest working frequency of NE564 can reach 50MHZ, which is powered by $+5 \mathrm{~V}$ single power supply. It is especially suitable for the FM signal of modulation and demodulation in high speed digital communication and 2FSK shift keying signal without external complex filter. CD4046 PLL is used in model RC voltage-controlled oscillator, which characteristic is a wide range of power supply voltage between $3 \mathrm{~V} 18 \mathrm{~V}$, dynamic power consumption is small, power consumption is only $600 \mathrm{~W}$ at the center frequency of F0 10kHzm and belongs to a micro power device

Secondly, we discuss the basic concepts and characteristics of FSK. Digital modulation refers to the process of using digital baseband signals to control the carrier and transform the digital baseband signals into digital band-pass signals (adjusted signals). Using the discrete values of digital signals, the technology of realizing digital modulation is called the key control method by opening the key control carrier. Digital frequency modulation is also called frequency shift keying (FSK - Frequency Shift Keying) and binary frequency shift keying is recorded as 2FSK. It is one of the key control methods, which uses the frequency change of the carrier to transmit digital information. The expression and waveform diagram of the 2FSK signal are shown as follows:

In the 2FSK signal, the frequency of the carrier changes with the binary baseband signal between the two frequency points of $1 \mathrm{~F}$ and $2 \mathrm{~F}$

$$
e_{2 F S K(t)}=\left\{\begin{array}{l}
A \cos \left(w_{1} t+\phi_{n}\right) \\
A \cos \left(w_{2} t+\theta_{n}\right)
\end{array}\right.
$$

If the binary sequence $\mathrm{s}(\mathrm{T})$ is 101001 , the waveform of the 2FSK signal is shown in the Fig3.

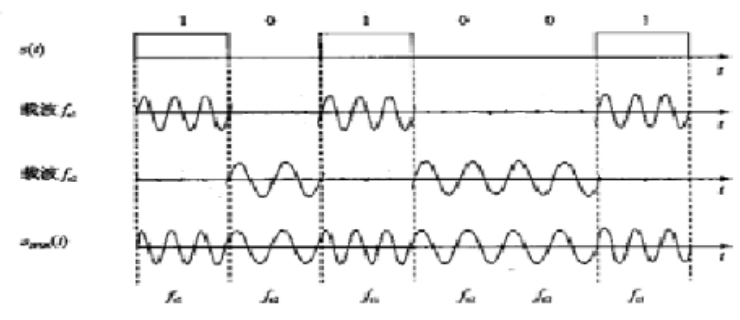

Fig 3 the waveform of 2FSK

Thirdly, we talk about the basic principle of FSK signal modulation. The digital frequency modulation signal can be divided into two cases, the phase continuity and the phase discrete. If the two signals are provided by different independent oscillator, the phase between them are not related. We usually name discrete phase digital FM signal. If two signals are provided by the same oscillation signal source, the phase of two carrier frequency is a continuous digital frequency modulation signal.

Then here is an introduction about the design of FSK signal modulation circuit by NE564. The circuit diagram is a FSK modulation circuit composed by NE564 and so on. In the circuit, NE564 is an analog PLL integrated circuit, which use two-state signal to control CD4016 and simulate FSK modulation switch. The two foot voltage of the NE564 is converted between $1.42 \mathrm{~V}$ to $5 \mathrm{~V}$ by CD4016.Therefore, in the case of continuous phase, the output of the voltage-controlled oscillator (VCO) can be changed. When the VCO center frequency capacitance is fixed, the offset between the FSK frequencies can be adjusted by $R_{1}$ and $R_{2}$.

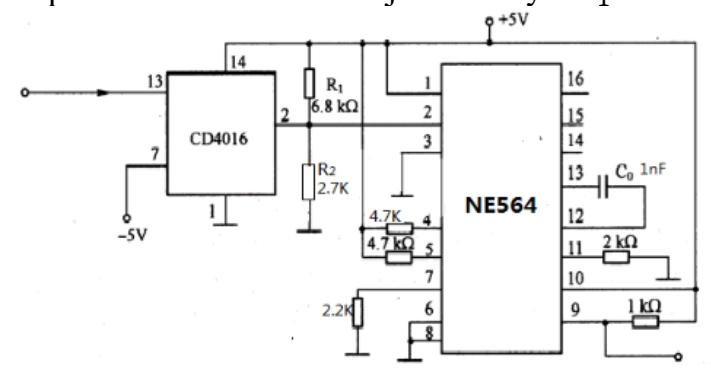

Fig 4 Demodulation of FSK signal by phase-locked loop chip

There are many ways to demodulate FSK signals. In this paper, we use the integrated phase-locked loop CD4046 to demodulate the FSK, which is shown in Fig4. When designing the phase-locked loop, we should fix the phase-locked loop on a carrier frequency 1F of FSK and output the high level and unlock the carrier frequency $2 \mathrm{~F}$ and output the low level. Then the output of phase locked loop filter can get baseband signal sequence. When inputting the FSK signal, PLL is locked. When inputting the frequency of signal, the low pass filter will output at high level. When inputting the frequency of signal, the low-pass filter will output at low level. Thus, we complete the demodulation of FSK signal. The principle block diagram of demodulation circuit of FSK signal is realized by CD4046. 


\section{CONCLUSION}

This paper gives a concise review of the PLL, as well as modulation and demodulation. The status of the PLL technology and its applications have been discussed. A summary of the PLL technology and its development tends are also included. In the future, I believe that the PLL will become more and more important and popular.

\section{REFERENCES}

1. Blanchard, Phase-Locked Loops: Application to Coherent Receiver Design. New York: Wiley, 1976.

2. F. M. Gardner, Phaselock Techniques, 2nd ed. New York: Wiley, 1979. [17] J. A. Afonso, A. J. Quiterio, and D. S. Arants, "A phase-locked loop with digital frequency compare for timing signal recovery," in Conf. Rec., 1979 Nat. Telecommun. Conf., paper 14.4 .

3. W. C. Lindsey and C. M. Chie, "A survey of digital phase-locked loops," Proc. IEEE, vol. 69, pp. 410431, Apr. 1981.
4. Zongsheng Lee, 2FSK modulation and demodulation circuit [J] (phase-locked loop NE564) $[\mathrm{J}]$. Shijia science and technology Career Technical College 2009:6 7

5. Hong Li, Demodulation of binary frequency shift keying signal based on CMOS integrated phaselocked loop $[\mathrm{J}]$. Guangdong University of Technology.2009:41 42.

6. Chongyi Zheng, The application of phase-locked loop in frequency modulation and demodulation circuit [J]. Hunan School of Building Materials.2003:36 37 .

7. Ricai Tian, Wengui Liu, Research on modulation and demodulation of FSK signal by CMOS PLL chip [J]. Harbin Institute of Technology.1996:65 67.

8. Yingna Guo, Design of a modulation and demodulation circuit for FSK signal [J]. Xi'an petroleum of University.2006:4 10 